\title{
EIN NEUER COPEPODE (GYCLOPOIDA: GNATHOSTOMA) VON DEN WESTINDISCHEN INSELN: HALICYCLOPS ANTIGUAENSIS N. SP.
}

\author{
von \\ HANS-VOLKMAR HERBST \\ Distelweg 1, 4150 Krefeld 29 - Hüls, Bundesrepublik Deutschland
}

\begin{abstract}
SUMMARY
In a well on the island of Antigua (West Indies), one female of a new species of Halicyclops was found, described as $H$. antiguaensis $\mathrm{n}$. sp. The male still remains unknown. The new species belongs to the thermophilus group of Halicyclops, characterized by a spiniform protuberance on each side of the genital somite, the end of which is curved backward, and a spine formula of the third exopodite segment of P1-P4 of 3, 4, 4, 3. It differs from $H$. thermophilus Kiefer, 1929, $H$. spinifer Kiefer, 1935, $H$. venezuelaensis Lindberg, 1954, and $H$. dedeckeri Brownell, 1983, by the relatively short spines on P5. The new species is closely related to $H$. latus Shen \& Tai, 1964, but in this species the furca and segment 3 of the 4th endopodite are relatively longer. Due to lack of information on the length of the furcal setae of the species of Shen \& Tai, further comparison is impossible.
\end{abstract}

\section{RÉSUMÉ}

Une femelle d'une nouvelle espèce de Halicyclops a été trouvée dans un puits de l'île Antigua (Indes Occidentales); elle est décrite comme $H$. antiguaensis n. sp., mais le mâle reste inconnu. La nouvelle espèce appartient au groupe d'espèces thermophilus, caractérisé par une protubérance spiniforme de chaque côté du somite génital, protubérance dont l'extrémité est courbée vers l'arrière, et par une formule 3, 4, 4, 3 des épines sur le 3ème segment de l'exopodite des P1-P4. L'espèce se distingue de $H$. thermophilus Kiefer, 1929, $H$. spinifer Kiefer, 1935, $H$. venezuelaensis Lindberg, 1954, et de $H$. dedeckeri Brownell, 1983, par les épines relativement courtes de P5. Elle est nettement apparentée à $H$. latus Shen \& Tai, 1964, mais la furca et le 3ème segment de l'endopodite 4 de cette der-

* Report 31 is published in the same issue of this journal. The fieldwork during which the material, described in the present paper, was collected, has been financially supported by the Beijerinck-Popping Fonds, Amsterdam, the Netherlands Foundation for the Advancement of Tropical Research (WOTRO), The Hague, and the Treub Maatschappij, Utrecht.

nière espèce sont relativement plus longs. La manque d'information sur la longueur des soies furcales de $H$. latus rend impossible une comparaison sur ce point.

\section{Halicyclops antiguaensis $\mathrm{n}$. sp.}

(Abb. 1-7)

Material. - Holotypus: 1 ९. Amsterdamer Expeditionen zu den Westindischen Inseln, Sta. 78-38, Antigua: Gemeinde St. Philip, Spencers, Comfort Hall, offene Quelle in sumpfigem Gebiet, ungefähr Meereshöhe; Abstand zur Küste ca. $2000 \mathrm{~m}$; Position $17^{\circ} 05^{\prime} 44^{\prime \prime} \mathrm{N} 61^{\circ}$ 42' 05 "W; 12 Apr. 1978 (Zoölogisch Museum Amsterdam).

Beschreibung. - Der Habitus des Weibchens (Abb. 1) zeigt die typische Halicyclops-Form mit breitem Thorax (Länge $=419 \mu \mathrm{m}$ ) sowie schmalerem und kürzerem Abdomen (Länge = $227 \mu \mathrm{m})$. Das Cephalothoraxsegment nimmt mehr als die halbe Länge des Thorax ein, in ihm liegt seine größte Breite mit $248 \mu \mathrm{m}$. Das 3. und 4. Thoraxsegment sind an den Seiten lappig nach hinten ausgezogen. Die LängenBreitenmaße der Abdominalsegmente sind:

Genitalsegment

2. Abdominalsegment

3. Abdominalsegment Analsegment

\begin{tabular}{crl} 
Länge & \multicolumn{2}{c}{ Breite } \\
78 & 101 & $\mu \mathrm{m}$ \\
71 & 66 & $\mu \mathrm{m}$ \\
37 & 61 & $\mu \mathrm{m}$ \\
26 & 58 & $\mu \mathrm{m}$
\end{tabular}

Das Genitalsegment (Abb. 2) ist mit einem Verhältnis von 0,78: 1 wesentlich breiter als lang, es trägt an seinen Außenrändern proximal von der Mitte, zwei nach hinten gerichtete, spitz auslaufende Anhänge, dahinter jeweils eine rundliche Vorwölbung. 


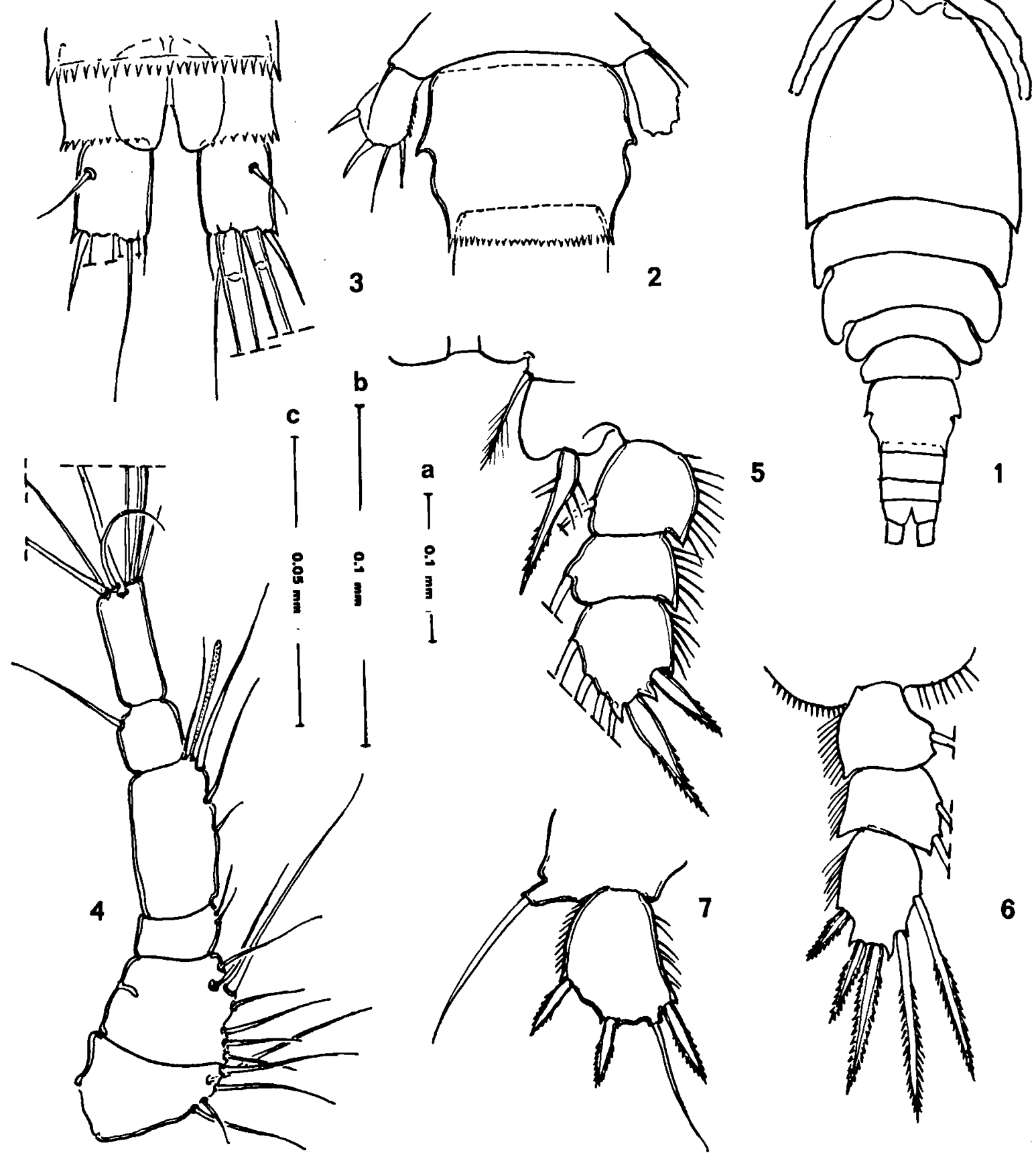

Halicyclops antiguaensis n. sp., \& Holotypus: 1, Habitus; 2, Genitalsegment; 3, Furka; 4, erste Antenne; 5, Endopodit 1; 6, Endopodit 4; 7, fünftes Bein.

Maßstab $a=$ Abb. 1; Maßstab b $=$ Abb. 2; Maßstab $c=$ Abb. 3-7. 
Die Hinterränder aller Abdominalsegmente sind dorsal mit einem gleichmäßig gezähnten Hyalinsaum versehen.

Die Furkaläste (Abb. 3) sind nur ein wenig länger als breit und rechteckig geformt; die Lateralrandborste inseriert am Ende des ersten Drittels des Seitenrandes und reicht bis zum Ende des Furkalastes. Von den Endborsten sind die beiden mittleren lang und kräftig ausgebildet, die laterale ist kurz und kräftig, die mediale sehr fein und erheblich kürzer als die laterale. Die Dorsalborste inseriert im medialen Bereich des Hinterrandes auf einer Vorwölbung und ist reichlich $1 \frac{1}{2} \mathrm{mal}$ so lang wie die laterale Endborste. Maße der Furka und ihrer Anhänge sind:

Länge: Breite $=(8+17): 20 \mu \mathrm{m}^{* *}$ (Verhältnis $=(0,40+0,85): 1)$; Apikalborsten von außen nach innen $=35: 208: 380: 14 \mu \mathrm{m}$ (Verhältnis auf die laterale $=1$ bezogen $=1: 5,94$ : 10,86: 0,40); Lateralrandborste $=20 \mu \mathrm{m}$ (Verhältnis s.o. $=0,57: 1)$; Dorsalborste $=56 \mu \mathrm{m}$ (Verhältnis s.o. $=1,60: 1$ ). Die beiden mittleren lang ausgebildeten Borsten sind befiedert, die übrigen sind unbefiedert.

Die sechsgliedrige 1. Antenne (Abb. 4) erreicht zurückgelegt nicht die Hälfte des Cephalothoraxsegments. Die dreigliedrige 2. Antenne und die Mundgliedmaßen zeigen keine spezifischen Unterschiede zu diesen in der Gattung übereinstimmend ausgebildeten Anhängen.

Die Dornformel der Außenastendglieder P1P4 ist 3, 4, 4, 3. Das 2. Basale des P1 (Abb. 5) trägt medial einen kräftigen, mit wenigen starken Fiedern besetzten Dorn, der etwas über das Ende des 2. Endopoditengliedes hinausreicht. Das Endglied Endopodit 4 (Abb. 6) ist relativ gedrungen und mit 5 Anhängen versehen, von denen die beiden des Medialrandes als kräftige, dornartig befiederte Borsten, die übrigen als Dornen zu bezeichnen sind. Maße des Endgliedes und seinen Anhänge sind:

Länge: Breite $=31: 23 \mu \mathrm{m}(=1,35: 1)$. Dorn des Lateralrandes $=17 \mu \mathrm{m}$, lateraler Enddorn $=24 \mu \mathrm{m}$, medialer Enddorn $=42 \mu \mathrm{m}$, distaler

** Das + Zeichen in der Längenangabe der Furka gibt die Insertion der Lateralrandborste an.
Dorn des Medialrandes $=49 \mu \mathrm{m}$ und proximaler Dorn des Medialrandes $=52 \mu \mathrm{m}$. Auf den Dorn des Lateralrandes $=1$ berechnet sind die Verhältnisse in der gleichen Reihenfolge $=$ 1: 1,41:2,47: 2,88: 3,06. Das Verhältnis von Gliedlänge: medialer Enddorn ist $=1,36: 1$.

Das Endglied des P5 (Abb. 7) ist etwa halbkreisförmig gestaltet, am Innen- und Außenrand locker bedornt und trägt 4 Anhänge: 3 Dornen und eine glatte Borste. Von dem mit dem 5. Thoraxsegment verschmolzenen Grundglied ist nur die Außenrandborste ( $=35$ $\mu \mathrm{m})$ erhalten. Maße des Endgliedes sind:

Länge: Breite $=36: 27 \mu \mathrm{m}(=1,35: 1)$. Dorn des Lateralrandes $=20 \mu \mathrm{m}$, lateraler Enddorn $=19 \mu \mathrm{m}$, mittlere Endborste $=43 \mu \mathrm{m}$, medialer Enddorn $=23 \mu \mathrm{m}$. Danach verhalten sich die Anhänge des Endgliedes auf den Dorn des Lateralrandes bezogen in der Reihenfolge von außen nach innen wie 1: 0,95: 2,15: 1,15. Das Verhältnis Gliedlänge: medialer Enddorn ist $=1,57: 1$.

Das Männchen ist noch unbekannt.

Verwandtschaftliche Beziehungen. Unter den Halicyclops-Arten mit der Dornformel $3,4,4,3$ besitzen außer der oben beschriebenen neuen Art die folgenden seitlich stehende, spitz auslaufende Anhänge am Genitalsegment: $H$. thermophilus Kiefer, 1929 (vergl. Heberer \& Kiefer, 1932), H. spinifer Kiefer, 1935, $H$. venezuelaensis Lindberg, 1954, $H$. latus Shen \& Tai, 1964, und $H$. dedeckeri Brownell, 1983.

Zur Kennzeichnung dieser Arten sollen die folgenden Verhältnismaße dienen, die z.T. nach den Abbildungen der Autoren berechnet werden mußten (Tabelle I). Brownell (1983) hat die innere Apikalborste der Furka nicht erkannt, dadurch sind die Dorsalborste mit der inneren Apikalborste und die Lateralrandborste mit der Dorsalborste verwechselt worden.

Nach den angegebenen Verhältnismaßen unterscheidet sich $H$. antiguaensis n. sp. von $H$. thermophilus durch die kürzere Furka, das kürzere Genitalsegment, einen im Verhältnis zum lateralen längeren medialen Enddorn am 
TABELLE I

Verhältnismaße verschiedener Halicyclops-Arten der thermophilus-Gruppe.

\begin{tabular}{|c|c|c|c|c|c|c|}
\hline & thermophilus 1 & spinifer ${ }^{2}$ & venezuelaensis & latus & dedeckeri & antiguaensis \\
\hline \multicolumn{7}{|l|}{ Genitalsegment } \\
\hline Länge: Breite & $0,93: 1$ & $0,80: 1$ & 1,17: 1 & $0,80: 1$ & 0,92: 1 & 0,78: 1 \\
\hline \multicolumn{7}{|l|}{ Furka } \\
\hline Länge: Breite & $1,5: 1$ & 1,23: 1 & $1: 1$ & $1,7: 1$ & $0,94: 1$ & $1,25: 1$ \\
\hline Endborsten & 1 & $1 \quad(1)$ & 1 & - & 1 & 1 \\
\hline auf die la- & 5,68 & $7,80(6,49)$ & 6,93 & - & 6,52 & 5,94 \\
\hline terale $=1$ & 13,68 & $13,77(12,06)$ & 16,14 & - & 12,73 & 10,86 \\
\hline berechnet & 0,44 & $0,50(0,45)$ & 0,57 & - & - & 0,40 \\
\hline Lateralrandborste & - & - & 0,64 & - & 0,84 & 0,57 \\
\hline Dorsalborste & 2,52 & - & 1,36 & - & 1,96 & 1,60 \\
\hline \multicolumn{7}{|l|}{ Endglied Endopodit 4} \\
\hline Länge: Breite & 1,35: 1 & 1,29: 1 & 1,47: 1 & $1,6: 1$ & 1,62: 1 & 1,35: 1 \\
\hline Länge: med. Enddorn & $1: 1,44$ & $1: 1,28$ & $1: 1,16$ & $1: 1,3$ & $1: 1,46$ & $1: 1,36$ \\
\hline med.: lat. Enddorn & 1,44: 1 & 1,36: 1 & 1,24: 1 & $1,8: 1$ & 1,52: 1 & 1,75: 1 \\
\hline \multicolumn{7}{|l|}{$P 5$} \\
\hline Länge: Breite & 1,35: 1 & 1,35: 1 & 1,59: 1 & 1,25: 1 & 1,48: 1 & 1,35: 1 \\
\hline Länge: med. Enddorn & 1,20: 1 & $0,84: 1$ & $0,58: 1$ & $2,0: 1$ & 1,19: 1 & 1,57: 1 \\
\hline Dorn Lateralrand $(=1)$ & 1 & 1 & 1 & 1 & 1 & 1 \\
\hline lat. Enddorn & 1 & 0,96 & 0,95 & 1 & 0,95 & 0,95 \\
\hline mittl. Endborste & 1,22 & 1,35 & 1,22 & 2,3 & 1,35 & 2,15 \\
\hline med. Enddorn & 1,11 & 1,19 & 1,62 & 1 & 1,17 & 1,15 \\
\hline
\end{tabular}

1 Maße für $H$. thermophilus nach Lindberg, 1952.

${ }^{2}$ Maße für $H$. spinifer nach Lindberg, 1941 (in Klammern Furkalborsten einer 2. Population).

Endglied Enp. 4 sowie eine erheblich längere mediale Endborste und einen kürzerem medialen Enddorn am Endglied des P5.

Gegen $H$. spinifer sind es die gleichen Merkmale am Endglied Endopodit 4 und P5.

$H$. venezuelaensis besitzt kürzere Furkaläste und ein längeres Genitalsegment als die neue Art. Außerdem ist der Längenunterschied zwischen medialem und lateralem Enddorn des Endgliedes Enp. 4 geringer. Der P5 besteht aus einem schlanken Glied, dessen medialer Enddorn extrem lang ausgebildet ist und noch die Länge der mittleren Endborste übertrifft. Dadurch unterscheidet sich $H$. venezuelaensis auch von allen bisher bekannten Arten der thermophilus-Gruppe.

$H$. latus dürfte die größte Ähnlichkeit mit der neuen Art besitzen, unterscheidet sich von ihr aber durch die längeren Furkaläste, das schlankere Endglied des Enp. 4 und die noch kürzeren Dornen am P5. Der Dorn am 2.
Basale des P1 ist bei $H$. latus kürzer. Durch die fehlenden Maßangaben über die Furkalborsten ist eine wichtige Vergleichsmöglichkeit entfallen. Das Genitalsegment scheint die gleiche Form wie bei $H$. antiguaensis zu besitzen. Im Text ist die 1. Antenne von $H$. latus irrtümlich als 8-gliedrig angegeben, sie ist aber richtig gezeichnet.

$H$. dedeckeri ist durch eine sehr kurze Furka, ein etwas längeres Genitalsegment, ein schlankeres Endglied Endopodit 4 und längere Dornen am P5 von der neuen Art unterschieden. Außerdem ist hier, abweichend von den übrigen Arten der thermophilus-Gruppe, der distale Anhang am Medialrand Endglied Endopodit 4 als lang gefiederte Borste ausgebildet.

Am Genitalsegment kommt die rundliche Vorwölbung hinter den dornartig ausgezogenen Seitenfortsätzen nur bei $H$. latus und $H$. antiguaensis $\mathrm{n}$. sp. vor. Die Zähnelung der distalen Hyalinmembran des 4. Abdominalsegments ist 
bei $H$. thermophilus, $H$. spinifer (vgl. Kiefer, 1936) und $H$. dedeckeri im mittleren Bereich etwas verlängert, für $H$. latus fehlen die Angaben, bei $H$. venezuelaensis und $H$. antiguaensis n. sp. sind keine Längenunterschiede der ventralen Zähnelung zu erkennen. Soweit Vergleichsmöglichkeiten bestehen, scheinen die beiden mittleren Endborsten der Furka bei $H$. antiguaensis n. sp. kürzer als bei den anderen Arten der thermophilus-Gruppe zu sein.

Nach den vorstehend gekennzeichneten Unterschieden zu den vergleichbaren Arten ist dem Halicyclops von der Insel Antigua spezifischer Rang zuzuerkennen.

\section{LITERATUR}

Brownell, C. L., 1983. A new euryhaline copepod from Cape Town: Halicyclops dedeckeri n. sp. (Copepoda: Cyclopoida). S. Afr. J. Zool., 18 (2): 62-66.
Heberer, G. F. Kiefer, 1932. Zur Kenntnis der Copepodenfauna der Sunda-Inseln. Arch. Naturgesch., (N.F.) 1: 225-274.

KIEFER, F., 1929. Neue Ruderfußkrebse von den SundaInseln. (1. Mitteilung über die Copepoden der Sunda-Expedition Rensch-Heberer.) Zool. Anz., 84 (1/4): 46-49.

- , 1935. Zur Kenntnis der Halicyclopen (Crustacea Copepoda). Zool. Anz., 110 (1/2): 10-13.

- - 1936. Freilebende Süß- und Salzwassercopepoden von der Insel Haiti. Arch. Hydrobiol., 30: 263-317.

LindBerg, K., 1941. Cyclopides (Crustacés Copépodes) de l'Inde, 5. Contribution à l'étude du genre Halicyclops Norman. K. fysiogr. Sällsk. Lund Förh., 19 (7): 98-121.

- - 1952. Cyclopides (Crustacés Copépodes) de Madagascar, 3. Mém. Inst. scient. Madagascar, (A) 7 (1): 53-67.

-_, 1954. Cyclopides (Crustacés Copépodes) de l'Amérique du Sud. Ark. Zool., (2) 7 (11): 193-222.

Shen Chia-jui \& Tai Ai-yun, 1964. Descriptions of new species of freshwater Copepoda from Kwangtung Province, South China. Acta zootax. sin., 1 (2): 367-396. 